\title{
Personalized Genomic Vaccine 001
}

National Cancer Institute

\section{Source}

National Cancer Institute. Personalized Genomic Vaccine 001. NCI Thesaurus. Code C136466.

A synthetic peptide-based, personalized cancer vaccine consisting of multiple patientspecific tumor peptides, which are immunogenic and unique to the patient's tumor, combined with the immunostimulant polyinosinic-polycytidylic acid stabilized with polylysine and carboxymethylcellulose (poly-ICLC), with potential immunomodulating and antineoplastic activities. Upon vaccination with the personalized genomic vaccine 001 (PGV001), the peptides stimulate the host immune system to mount a specific and potent cytotoxic T-lymphocyte (CTL) response ag ainst tumor cells expressing the neoantigens, which results in tumor cell lysis. The adjuvant Poly-ICLC is added to boost the immune response to the peptides and together will expand immune cells to target cancer. The adjuvant poly-ICLC is a ligand for toll-like receptor-3 (TLR3) and induces the release of cytokines which may help to boost the immune response against the selected neoantigens. 